\title{
ISOLAMENTO E AVALIAÇÃO DO POTENCIAL CITOTÓXICO DE DERIVADOS FENÓLICOS DE Schinus terebinthifolius Raddi (Anacardiaceae)"
}

\author{
Jeferson S. Santana, Patricia Sartorelli e João Henrique G. Lago*
}

Instituto de Ciências Ambientais, Químicas e Farmacêuticas, Universidade Federal de São Paulo, 09972-270 Diadema - SP, Brasil Alisson L. Matsuo

Departamento de Micro, Imuno e Parasitologia, Universidade Federal de São Paulo, 04023-062 São Paulo - SP, Brasil

Recebido em 27/4/12; aceito em 1/8/12; publicado na web em 25/9/12

\begin{abstract}
ISOLATION AND EVALUATION OF CYTOTOXIC POTENTIAL OF PHENOLIC DERIVATIVES FROM Schinus terebinthifolius Raddi (Anacardiaceae). The EtOH extract from leaves of S. terebinthifolius was subjected to partition between EtOH: $\mathrm{H}_{2} \mathrm{O}$ and hexane, $\mathrm{CH}_{2} \mathrm{Cl}_{2}$, and EtOAc. The phases obtained were evaluated in vitro against human tumoral cell lines and the EtOAc phase exhibited activity. Chromatographic procedures afforded gallic acid (1), methyl (2) and ethyl (3) gallates, trans-catechin (4), quercitrin (5), and afzelin (6), being the first occurrence of 1, 4 and $\mathbf{6}$ in S. terebinthifolius. In vitro cytotoxic evaluation of $\mathbf{1}-\mathbf{6}$ indicated that gallic acid (1) displayed higher activity than ethyl gallate (3) against HL-60 and HeLa cells, while compounds 2, 4 - 6 were inactive.
\end{abstract}

Keywords: Schinus terebinthifolius; phenolic derivatives; cytotoxic activity.

\section{INTRODUÇÃO}

Schinus terebinthifolius Raddi (Anacardiaceae) é uma árvore monoica de tamanho médio, com ocorrência em todo o território brasileiro, principalmente em regiões da Mata Atlântica. ${ }^{1,2} \mathrm{Na}$ medicina popular, esta planta tem sido usada no tratamento de úlceras, problemas respiratórios, feridas, reumatismo, gota, diarreia, doenças de pele e artrite, ${ }^{3}$ bem como antisséptico e anti-inflamatório. ${ }^{4}$ Além disso, a decocção de flores, caules, frutos e folhas é utilizada para o tratamento de tumores e lepra. ${ }^{5}$

Fitoquimicamente, esta espécie produz ácidos graxos e terpenoides, principalmente os derivados ácidos $3 \alpha$-masticadienoico (schinol) e masticadienoico, ${ }^{6}$ os quais apresentam atividade inibitória da fosfolipase $\mathrm{A} 2^{7}$ além de potencial fungitóxico frente a Paracoccidioides brasiliensis. ${ }^{8}$ Derivados fenólicos, tais como os galatos de metila e etila além de flavonoides (miricetina, miricetrina e quercitrina) foram isolados do extrato polar das folhas, cuja ocorrência foi associada à elevada atividade antirradicalar. ${ }^{9}$ Adicionalmente, a análise química das cascas do tronco mostrou a existência de antraquinonas, xantonas e esteroides livres. ${ }^{10}$ Os óleos essenciais de folhas, flores e frutos de $S$. terebinthifolius de diferentes regiões foram previamente analisados, os quais se mostraram compostos basicamente por derivados mono e sesquiterpênicos. ${ }^{11}$ No que se refere à avaliação da atividade biológica, os óleos voláteis dos frutos foram analisados e apresentaram atividade alelopática, ${ }^{12}$ citotóxica ${ }^{13}$ e tripanocida, ${ }^{14}$ enquanto que o óleo das folhas mostrou atividade citotóxica. ${ }^{15}$

Como parte de nossos estudos visando o reconhecimento de compostos com ação citotóxica em espécies vegetais brasileiras, neste trabalho foi realizado o estudo fitoquímico do extrato etanólico das folhas de S. terebinthifolius, o qual apresentou potencial in vitro frente a células tumorais humanas de melanoma (A2058), adenocarcinoma de mama (MCF7), leucemia (HL-60) e carcinoma cervical (HeLa). Após sucessivos processos de partição e purificação em gel de Sephadex LH-20 e em sílica, foi possível o isolamento do ácido gálico (1), dos galatos de metila (2) e etila (3) e dos flavonoides trans-catequina (4), quercitrina (5) e afzelina (6), cuja identificação

*e-mail: joao.lago@unifesp.br

\#Artigo em homenagem ao Prof. Otto R. Gottlieb (31/8/1920-19/6/2011) estrutural foi realizada através da análise dos dados espectrométricos, principalmente $\mathrm{RMN}$ de ${ }^{1} \mathrm{H}$ e de ${ }^{13} \mathrm{C}$.

\section{PARTE EXPERIMENTAL}

\section{Instrumentação e procedimentos gerais}

As separações cromatográficas em coluna foram realizadas em gel de sílica (Merck, 230-400 mesh) e em Sephadex LH-20 (Amersham Biosciences), enquanto que as separações por cromatografia em camada delgada foram realizadas em gel de sílica $60 \mathrm{PF}_{254}$ (Merck). Para as análises por cromatografia líquida de alta eficiência foi utilizado um equipamento Dionex (modelo Ultimate 3000) munido de coluna Luna (5 $\mu$-C18 de $250 \mathrm{~mm}$ de comprimento $\mathrm{x} 4,60 \mathrm{~mm}$ de d.i. para sistema analítico e de $250 \mathrm{~mm}$ de comprimento $\mathrm{x} 10,0 \mathrm{~mm}$ de d.i. para sistema semipreparativo), de detector espectrofotométrico (modelo UVD-17OU) e de bomba quaternária. Os espectros de RMN foram registrados em espectrômetros Bruker, modelos AC-200 e DPX-300 operando, respectivamente, a 200 e $300 \mathrm{MHz}$ para o núcleo do hidrogênio e a 50 e $75 \mathrm{MHz}$ para o núcleo do carbono-13. Dimetilsulfóxido-d $\mathrm{d}_{6}$ (Tedia Brazil) e metanol- $\mathrm{d}_{4}$ (Tedia Brazil) foram usados como solventes, sendo o pico residual do derivado não deuterado empregado como padrão interno.

\section{Material vegetal}

O material vegetal foi coletado em fevereiro de 2010, de um espécime localizado na cidade de Mogi-Guaçú/São Paulo. A identificação botânica foi realizada pela Profa. Dra. M. C. M. Young, sendo que a exsicata preparada com o espécime estudado foi comparada com aquela depositada previamente no Instituto de Botânica de São Paulo (SP272591).

\section{Extração e isolamento das substâncias}

As folhas de $S$. terebinthifolius foram secadas em estufa a $40{ }^{\circ} \mathrm{C}$ e submetidas à moagem. Deste processo, foi obtido $1 \mathrm{~kg}$ do material vegetal que foi extraído com hexano até o esgotamento para retirada do material graxo. Na sequência, a torta foi submetida à extração 
exaustiva com EtOH à temperatura ambiente. A destilação do solvente sob pressão reduzida forneceu o extrato etanólico bruto, que foi avaliado quanto ao potencial citotóxico in vitro. $\mathrm{O}$ extrato EtOH foi submetido à partição entre EtOH: $\mathrm{H}_{2} \mathrm{O}$ 1:2 (500 mL) e hexano, $\mathrm{CH}_{2} \mathrm{Cl}_{2}$ e AcOEt (4 X $250 \mathrm{~mL}$ cada solvente), sucessivamente, sendo que foi observada atividade citotóxica na fase em AcOEt. Desta forma, parte da fase em AcOEt ( $3 \mathrm{~g}$ ) foi submetida a fracionamento cromatográfico em gel de Sephadex LH-20 (30 X 2 cm) eluída com $\mathrm{MeOH}$, sendo aplicados $300 \mathrm{mg}$ de material por vez. Em cada um desses procedimentos, foram obtidas 60 frações $(6 \mathrm{~mL}$ cada) que, após análise conjunta por $\mathrm{CCD}$, foram reunidas em 6 grupos $(\mathrm{A}-\mathrm{F})$. O grupo B (1242 mg) foi submetido ao fracionamento cromatográfico em gel de sílica eluído com misturas de $\mathrm{CHCl}_{3}: \mathrm{MeOH}$ em polaridade crescente, fornecendo 57 frações $(10 \mathrm{~mL}$ cada) que, após análise por $\mathrm{CCD}$, foram reunidas em 5 grupos (B-1 - B-5), dos quais B-1 (1076 $\mathrm{mg}$ ) e B-3 (27 mg) estavam puros e constituídos por $\mathbf{2}$ e 3, respectivamente. O grupo C (249 mg) foi submetido à separação em gel de Sephadex LH-20 (28 X 2 cm) eluído com MeOH, fornecendo 21 frações $(10 \mathrm{~mL})$ que, após monitoramento por $\mathrm{CCD}$, foram reunidas em 5 grupos (C-1 - C-5). Deste processo, foram obtidos $11 \mathrm{mg}$ de 4 do grupo C-1 e $4 \mathrm{mg}$ de 1 do grupo C-5. A fração C-4 (61 mg) foi purificada através de CLAE semipreparativo (MeOH: $\mathrm{H}_{2} \mathrm{O}$ 75:15), possibilitando o isolamento de de $\mathbf{5}(13 \mathrm{mg})$ e $\mathbf{6}(8 \mathrm{mg})$.

\section{Ácido gálico (1)}

Sólido branco amorfo. RMN ${ }^{1} \mathrm{H}\left(200 \mathrm{MHz}, \mathrm{CD}_{3} \mathrm{OD}\right) \delta_{\mathrm{H}}$ (mult.; $\mathrm{H}): 6,92(s ; \mathrm{H}-2 / \mathrm{H}-6), 9,36(s ; \mathrm{OH}) . \mathrm{RMN}{ }^{13} \mathrm{C}\left(50 \mathrm{MHz}, \mathrm{CD}_{3} \mathrm{OD}\right)$ $\delta_{\mathrm{C}}: 120,9$ (C-1), 109,5 (C-2/C-6), 145,8 (C-3/C-5), 138,4 (C-4), $167,9(\mathrm{C}-7)$.

\section{Galato de metila (2)}

Sólido branco amorfo. RMN ${ }^{1} \mathrm{H}\left(300 \mathrm{MHz}, \mathrm{CD}_{3} \mathrm{OD}\right) \delta_{\mathrm{H}}$ (mult.; $\mathrm{H}): 7,04(s ; \mathrm{H}-2 / \mathrm{H}-6), 3,80(s ; \mathrm{H}-8) . \mathrm{RMN}^{13} \mathrm{C}\left(75 \mathrm{MHz}, \mathrm{CDCl}_{3}\right) \delta_{\mathrm{C}}$ : 124,0 (C-1), 110,2 (C-2/C-6), 146,6 (C-3/C-5), 123,7 (C-4), 169,1 (C-7), 52,4 (C-8).

\section{Galato de etila (3)}

Sólido branco amorfo. $\mathrm{RMN}^{1} \mathrm{H}\left(300 \mathrm{MHz}, \mathrm{CD}_{3} \mathrm{OD}\right) \delta_{\mathrm{H}}$ (mult.; $J$ em Hz; H): 7,02 ( $s$; H-2/H-6), 3,60 ( $q$; 7,2; H-8), 1,17 ( $t ; 7,2 ; \mathrm{H}-9)$. RMN ${ }^{13} \mathrm{C}\left(75 \mathrm{MHz}, \mathrm{DMSO}-\mathrm{d}_{6}\right) \delta_{\mathrm{C}}: 119,6$ (C-1), 108,5 (C-2/C-6), 145,6 (C-3/C-5), 138,6 (C-4), 165,9 (C-7), 60,0 (C-8), 14,3 (C-9).

\section{Trans-catequina $(4)$}

Sólido amarelo amorfo. RMN ${ }^{1} \mathrm{H}\left(300 \mathrm{MHz}, \mathrm{CD}_{3} \mathrm{OD}\right) \delta_{\mathrm{H}}$ (mult.; $J$ em Hz; H): 4,56 ( $d$; 7,5; H-2), 3,97 (ddd; 7,5, 8,0 e 5,4; H-3), 2,84 $(d d, 16,2$ e 5,$4 ; \mathrm{H}-4 \mathrm{a}), 2,50$ ( $d d ; 16,2$ e 8,$0 ; \mathrm{H}-4 \mathrm{~b}), 5,85$ ( $d ; 2,1 ; \mathrm{H}-6)$, 5,93 ( $d ; 2,1 ; \mathrm{H}-8), 6,84\left(d ; 1,8 ; \mathrm{H}-2^{\prime}\right), 6,77$ ( $\left.d ; 8,1 ; \mathrm{H}^{-} 5^{\prime}\right), 6,71$ ( $d d ; 8,1$ e 1,$\left.8 ; \mathrm{H}^{\prime} 6^{\prime}\right)$. RMN ${ }^{13} \mathrm{C}\left(75 \mathrm{MHz}, \mathrm{CD}_{3} \mathrm{OD}\right) \delta_{\mathrm{C}}: 82,9$ (C-2), 68,8 (C-3), 28,6 (C-4), 157,8 (C-5), 96,4 (C-6), 157,3 (C-7), 95,6 (C-8), 157,0 (C-9), 100,9 (C-10), 132,7 (C-1'), 115,3 (C-2'), 146,3 (C-3'/C-4'), $116,2\left(\mathrm{C}-5^{\prime}\right), 120,1$ (C-6').

\section{Quercitrina (5)}

Sólido branco amorfo. RMN ${ }^{1} \mathrm{H}\left(300 \mathrm{MHz}, \mathrm{CD}_{3} \mathrm{OD}\right) \delta_{\mathrm{H}}$ (mult.; $J$ em Hz; H): 6,37 (d;2,1; H-6), 6,19 (d; 2,1; H-8), 7,34 (d; 2,1; H-2'); 6,94 (d; 8,7; H-5'), 7,30 (dd; 8,7 e 2,1; H-6'), 5,34 (d; 1,5; H-1"), 3,32 - 4,23 (m; H-2" a H-5"); 0,94 (d; 6,0; H-6"). RMN ${ }^{13} \mathrm{C}$ (75 MHz, CD 3 OD) $\delta_{\mathrm{C}}: 159,4$ (C-2), 136,3 (C-3), 179,7 (C-4), 163,3 (C-5), 99,9 (C-6), 165,9 (C-7), 94,9 (C-8), 158,6 (C-9), 106,1 (C10), 123,0 (C-1'), 117,1 (C-2'), 146,5 (C-3'), 149,9 (C-4'), 116,5 (C-5'), 123,1 (C-6'), 103,6 (C-1"), 72,1 (C-2"/C3”), 73,3 (C-4”), 72,0 (C-5"), 17,8 (C-6").

\section{Afzelina (6)}

Sólido branco amorfo. RMN ${ }^{1} \mathrm{H}\left(300 \mathrm{MHz}, \mathrm{CD}_{3} \mathrm{OD}\right) \delta_{\mathrm{H}}$ (mult.; $J$ em Hz; H): 6,37 (d; 2,1; H-6), 6,19 (d; 2,1; H-8), 7,74 (d; 8,7; H-2'/H-6'); 6,94 (d; 8,7; H-3'/H-5'), 5,37 (d; 1,5; H-1'), 3,41 - 4,23 ( $m$; H-2" a H-5"), 0,92 (d; 5,4; H-6"). RMN ${ }^{13} \mathrm{C}$ (75 MHz, CD $\left.3 \mathrm{OD}\right)$ $\delta_{\mathrm{C}}: 159,4$ (C-2), 136,3 (C-3), 179,7 (C-4), 163,3 (C-5), 99,9 (C-6), 165,9 (C-7), 94,8 (C-8), 158,6 (C-9), 106,1 (C-10), 122,7 (C-1'), 132,0 (C-2'/C-6'), 116,6 (C-3'/C-5'), 161,7 (C-4'), 103,6 (C-1”), 72,1 (C-2"/C3”), 73,4 (C-4”), 72,3 (C-5”), 17,8 (C-6”).

\section{Linhagens celulares}

As linhagens celulares de melanoma humano (A2058), adenocarcinoma da mama (MCF7) e leucemia (HL-60) foram fornecidas pelo Instituto Ludwig de Pesquisa sobre o Câncer, enquanto que as linhagens de carcinoma cervical humano (HeLa) foram fornecidas pelo Dr. H. P. Monteiro, da UNIFESP.

Todas as células foram cultivadas a $37^{\circ} \mathrm{C} \mathrm{em}$ atmosfera umidificada contendo 5\% de $\mathrm{CO}_{2}$, em meio RPMI 1640 (Invitrogen, Carlsbad, CA) suplementado com $10 \mathrm{mM}$ de $\mathrm{N}$-2-hidroxietilpiperazina- $\mathrm{N}_{2}$ etanossulfónico (HEPES) (Sigma, St. Louis, MO), bicarbonato de sódio $24 \mathrm{mM}$ (Sigma), $40 \mathrm{mg} / \mathrm{L}$ de gentamicina (Schering-Plough, São Paulo, Brasil), pH 7,2 e 10\% de soro fetal bovino (Invitrogen).

\section{Avaliação da atividade citotóxica in vitro}

As substâncias isoladas $\mathbf{1}$ - $\mathbf{6}$ foram ressuspendidas individualmente em dimetilsulfóxido (DMSO) em concentrações que variaram entre $0-100 \mu \mathrm{g} / \mathrm{mL}$, em triplicata. Na sequência, a solução foi diluída em meio RPMI contendo soro fetal bovino a $10 \%$ e incubada com 1 $\times 10^{4}$ células em uma placa de 96 poços. Após 24 h de incubação, a viabilidade das células foi medida utilizando-se o Kit de Proliferação Celular I (Sigma), um ensaio colorimétrico baseado na redução do MTT. ${ }^{16}$ DMSO e cisplatina foram usados como controles negativo e positivo, respectivamente. As leituras foram feitas em um leitor de placas a $570 \mathrm{~nm}$ com uma referência de $650 \mathrm{~nm}$. Os valores de $\mathrm{CI}_{50}$ $(\mu \mathrm{g} / \mathrm{mL})$ foram calculados a partir de regressão não linear utilizando o programa Prisma - GraphPad Software.

\section{RESULTADOS E DISCUSSÃO}

O extrato etanólico das folhas de S. terebinthifolius apresentou potencial in vitro frente a células de melanoma humano (A2058), adenocarcinoma da mama (MCF7), leucemia (HL-60) e carcinoma cervical (HeLa). O processo de isolamento dos constituintes do extrato ativo incluiu partição sequencial com hexano, $\mathrm{CH}_{2} \mathrm{Cl}_{2}$ e $\mathrm{AcOEt}$ seguida de fracionamento cromatográfico através de permeação em gel de sílica e/ou Sephadex LH-20, bem como CLAE semipreparativa o que resultou no isolamento de seis compostos fenólicos (1-6, Figura 1), cujas estruturas foram identificadas com base na análise dos dados obtidos dos espectros de RMN.

Os compostos 1-3 foram caracterizados, respectivamente, como ácido gálico e galatos de metila e de etila pela comparação dos dados de RMN com os descritos na literatura. ${ }^{17}$ Como discutido em comunicação anterior, ${ }^{9}$ a possibilidade dos compostos $\mathbf{2}$ e $\mathbf{3}$ serem artefatos devido aos solventes utilizados nos processos de extração e fracionamento foi descartada quando uma pequena parte do material vegetal foi extraído diretamente com $\mathrm{MeOH}$. Apesar dos compostos 2 e $\mathbf{3}$ terem sido detectados anteriormente em $\mathrm{S}$. terebinthifolius, ${ }^{9}$ essa é a primeira descrição do ácido gálico livre nessa espécie vegetal.

$\mathrm{O}$ espectro de RMN de ${ }^{1} \mathrm{H}$ de $\mathbf{4}$ mostrou um sinal referente a hidrogênio ligado a carbono carbinólico em $\delta 3,97$ ( $d d d, J=7,5 ; 8,0$ e $5,4 \mathrm{~Hz}, \mathrm{H}-3$ ) acoplando com hidrogênio ligado a carbono oxibenzílico 
<smiles>[R]OC(=O)c1cc(O)c(O)c(O)c1</smiles><smiles>Oc1cc(O)c2c(c1)O[C@H](c1ccc(O)c(O)c1)[C@H](O)C2</smiles>

4<smiles>O=c1c(O)c(-c2ccc(O)c(O)c2)oc2cc(O)cc(O)c12</smiles>

5
6<smiles>COc1c(-c2ccc(O)cc2)oc2cc(O)cc(O)c2c1=O</smiles>

Figura 1. Estrutura dos metabólitos 1-6 isolados de S. terebinthifolius

em $\delta 4,56(d, J=7,5 \mathrm{~Hz}, \mathrm{H}-2)$. Esses sinais, quando associados àqueles relativos aos hidrogênios de anel aromático entre $\delta$ 5,8-6,8 e aos atribuídos a H-4 em $\delta 2,84(d d, J=16,2$ e $5,4 \mathrm{~Hz})$ e em $\delta 2,50$ $(d d, J=16,2$ e $8,0 \mathrm{~Hz}$ ), permitiram inferir a ocorrência da catequina, cuja configuração entre $\mathrm{C}-2 / \mathrm{C}-3$ foi definida como trans através da constante de acoplamento de 7,5 Hz do dupleto de $\mathrm{H}-2$. A estrutura da trans-catequina foi confirmada pela análise do espectro de RMN de ${ }^{13} \mathrm{C}$, devido aos sinais em $\delta 82,9(\mathrm{C}-2), 68,8$ (C-3) e 28,6 (C-4), além daqueles entre $\delta$ 95-160 referentes aos carbonos aromáticos, seguida da comparação com dados descritos na literatura, ${ }^{18}$ sendo esta a primeira ocorrência de $\mathbf{4} \mathrm{em}$ S. terebinthifolius.

Os espectros de RMN de ${ }^{1} \mathrm{H}$ das substâncias $\mathbf{5}$ e $\mathbf{6}$ mostraram sinais característicos de flavonoides glicosilados, devido aos sinais relativos à ramnose em $\delta 5,34 / 5,37(d, J=1,5 \mathrm{~Hz}, \mathrm{H}-1$ "'), 3,3 - 4,2 (H-2" a H-5") e em $\delta 0,94 / 0,92$ ( $d, J \sim 6 \mathrm{~Hz}, \mathrm{H}-6$ ") e dos dupletos em $\delta 6,37$ $(J=1,8 \mathrm{~Hz}, \mathrm{H}-6), 6,19(J=2,1 \mathrm{~Hz}, \mathrm{H}-8)$, relativos aos hidrogênios do anel aromático A. A diferença entre os dois compostos foi observada no anel $\mathrm{B}$, devido aos sinais relativos a três hidrogênios em $\delta 7,34$ ( $d, J=2,1 \mathrm{~Hz}, \mathrm{H}-2^{\prime}$ '), 6,94 ( $d, J=8,7 \mathrm{~Hz}, \mathrm{H}-5$ ') e 7,30 ( $d d, J=8,4$ e $2,1 \mathrm{~Hz}, \mathrm{H}-6$ ') no espectro de $\mathbf{5}$ e a quatro hidrogênios em $\delta 7,74(d$, $J=8,7 \mathrm{~Hz}, \mathrm{H}-2^{\prime} / \mathrm{H6}^{\prime}$ ), 6,94 (d, $J=8,7 \mathrm{~Hz}, \mathrm{H}-3^{\prime} / \mathrm{H}-5^{\prime}$ ') no espectro de 6. Finalmente, a comparação dos dados de $\mathrm{RMN}$ de ${ }^{13} \mathrm{C}$ registrados com aqueles descritos na literatura ${ }^{19}$ permitiu inferir que as estruturas dos derivados 5 e $\mathbf{6}$ correspondem aos flavonoides quercetina-3-O- $\beta$-ramnosídeo (quercitrina) e canferol-3-O- $\beta$-ramnosídeo (afzelina). O composto 5 foi detectado previamente em S. terebinthifolius, ${ }^{9}$ enquanto que esse é o primeiro relato de $\mathbf{6}$ nesta espécie.

A investigação fitoquímica do extrato polar das folhas de $S$. terebinthifolius resultou no isolamento de diversos derivados fenólicos, com ênfase nos derivados do ácido gálico além de flavonoides glicosilados, de acordo com o observado em trabalho anterior com essa espécie. ${ }^{9}$ No entanto, é interessante observar que não foi verificada a ocorrência de derivados da miricetina, um flavonol tri-hidroxilado no anel $\mathrm{B}^{9}$ formado a partir dos mesmos precursores que geram o ácido gálico, mas a presença de flavonoides de ocorrência menos restrita (5 e 6), cujos derivados aglicônicos (quercetina e canferol) foram isolados anteriormente dos galhos de S. terebinthifolius. ${ }^{8}$

Uma vez que o extrato em EtOH mostrou potencial frente a diferentes linhagens tumorais humanas in vitro, esse foi submetido a um processo de partição líquido/líquido sendo que a fase obtida em AcOEt apresentou atividade. As substâncias isoladas (1-6) foram também avaliadas, porém o ácido gálico (1) foi o mais ativo, principalmente para células de leucemia humana (HL-60) e de carcinoma humano (HeLa) com valores de $\mathrm{CI}_{50}$ de $2,4 \pm 0,1$ e 13,8 $\pm 0,7 \mu \mathrm{g} /$ $\mathrm{mL}$, respectivamente, sendo inferiores aos calculados para o controle positivo cisplatina (Tabela 1).

Tabela 1. Valores* de $\mathrm{CI}_{50}(\mu \mathrm{g} / \mathrm{mL})$ para os derivados isolados de S. terebinthifolius em ensaios in vitro frente a linhagens tumorais humanas

\begin{tabular}{ccccc}
\hline Substâncias & HeLa & MCF7 & A2058 & HL-60 \\
\hline $\mathbf{1}$ & $13,8 \pm 0,7$ & $22 \pm 1$ & $47 \pm 2$ & $2,4 \pm 0,1$ \\
$\mathbf{2}$ & $>100$ & $>100$ & $>100$ & $>100$ \\
$\mathbf{3}$ & $53,9 \pm 0,5$ & $>100$ & $94 \pm 1$ & $6,8 \pm 0,3$ \\
$\mathbf{4}$ & $>100$ & $>100$ & $>100$ & $>100$ \\
$\mathbf{5}$ & $>100$ & $>100$ & $>100$ & $74,63 \pm 0,07$ \\
$\mathbf{6}$ & $>100$ & $>100$ & $>100$ & $>100$ \\
Cisplatina & $20 \pm 1$ & nd & nd & $21 \pm 2$ \\
\hline
\end{tabular}

*média de 3 experimentos independentes; nd: não determinado

Por outro lado, o composto 3 mostrou atividade para as mesmas linhagens HL-60 e HeLa com valores de $\mathrm{CI}_{50}$ de 6,8 \pm 0,3 e 53,9 \pm 0,5 $\mu \mathrm{g} / \mathrm{mL}$, respectivamente, enquanto que o derivado esterificado 2 não mostrou atividade $\left(\mathrm{CI}_{50}>100 \mu \mathrm{g} / \mathrm{mL}\right)$. Tais dados indicam que a presença do grupo carboxílico livre é crucial para a atividade observada.

Finalmente, uma vez que os flavonoides 4-6 também se mostraram inativos nos ensaios realizados, os dados obtidos sugerem que o potencial observado para a fase em AcOEt do extrato etanólico das folhas de $S$. terebinthifolius se deva, pelo menos em parte, à presença do composto 1 , mesmo em pequena concentração $(0,075 \%)$. A atividade antitumoral in vitro do ácido gálico frente a diferentes linhagens celulares foi descrita anteriormente, ${ }^{20}$ porém essa é a primeira descrição da presença desse composto no extrato ativo de $S$. terebinthifolius.

\section{AGRADECIMENTOS}

Ao programa BIOTA/FAPESP pelo apoio financeiro (projeto número 11/51739) e ao CNPq e à FADA/UNIFESP pelas bolsas de produtividade à pesquisa.

\section{REFERÊNCIAS}

1. Corrêa, M. P.; Dicionário de Plantas Úteis do Brasil e das Exóticas Cultivadas, Imprensa Nacional: Rio de Janeiro, 1984, vol. 1.

2. Lorenzi, H.; Árvores Brasileiras: manual de identificação e cultivo de plantas arbóreas nativas do Brasil, Ed. Plantarum: São Paulo, 1992.

3. Morton, J. F.; Economic Bot. 1978, 32, 353.

4. Medeiros, K. C. P.; Monteiro, J. C.; Diniz, M. F. F. M.; Medeiros, I. A.; Silva, B. A.; Piuvezam, M. R.; Rev. Bras. Farmacogn. 2007, 17, 23.

5. Schmourlo, G.; Mendonça-Filho, R. R.; Alviano, C. S.; Costa, S. S.; J. Ethnopharmacol. 2005, 96, 563.

6. Moneam, N. M. A.; Ghoneim, T.; J. Chromatogr., A 1986, 361, 391; Lloyd, H. A.; Jaouni, T. M.; Evans, S. L.; Morton, J. F.; Phytochemistry 1977, 16, 1301; Campello, J. P.; Marsaioli, A. J.; Phytochemistry 1975, 14, 2300; Campello, J. P.; Marsaioli, A. J.; Phytochemistry 1974, 13, 659.

7. Jain, M. K.; Yu, B. Z.; Rogers, J. M.; Smith, A. E.; Boger, E. T. A.; Ostrander, R. L.; Rheingold, A. L.; Phytochemistry 1995, 39, 537.

8. Johann, S.; Sá, N. P.; Lima, L. A. R. S.; Cisalpino, P. S.; Cota, B. B.; Alves, T. M. A.; Siqueira, E. P.; Zani, C. L.; Ann. Clin. Microbiol. Antimicrob. 2010, 9, 30. 
9. Ceruks, M.; Romoff, P.; Fávero, O. A.; Lago, J. H. G.; Quim. Nova 2007, 30, 597.

10. Lima, M. R. F.; Luna, J. S.; Santos, A. F.; Andrade, M. C. C.; Sant'ana, A. E. G.; Genet, J. P.; Marquez, B.; Neuville, L.; Moreau, N.; J. Ethnopharmacol. 2006, 105, 137.

11. Ibrahim, M. T.; Fobbe, R.; Nolte; J.; Bull. Fac. Pharm. 2004, 42, 289; Singh, A. K.; Gupta, K. C.; Brophy, J. J.; J. Essent. Oil Res. 1998, 10, 697; Jamal, Y.; Agusta, A.; Maj. Farm. Indo. 2001, 12, 135; Chowdhury, A. R.; Tripathi, S.; Ind. Perfum. 2001, 45, 257; Malik, M. S.; Mahmud, S.; Sattar, A.; Pak. Sci. Int. (Lahore) 1994, 6, 351.

12. Barbosa, L. C. A.; Demuner, A. J.; Clemente, A. D.; Paula, V. F.; Ismail, F. M. D.; Quim. Nova 2007, 30, 1959.

13. Bendaoud, H.; Romdhane, M.; Souchard, J. P.; Cazaux, S.; Bouajila, J.; J. Food Sci. 2010, 75, C466.

14. Sartorelli, P.; Santana, J. S.; Guadagnin, R. C.; Lago, J. H. G.; Pinto, E. G.; Tempone, A. G.; Stefani, H. A.; Soares, M. G.; Silva, A. M.; Quim. Nova 2012, 35, 743.

15. Santana, J. S.; Sartorelli, P.; Guadagnin, R. C.; Matsuo, A. L.; Figueiredo, C. R.; Soares, M. G.; Zanin, J. L. B.; Silva, A. M.; Lago, J. H. G.; Pharm. Biol. 2012, 50, 1248.
16. Matsuo, A. L.; Figueiredo, C. R.; Arruda, D. C.; Pereira, F. V.; Scutti, J. A. B.; Massaoka, M. H.; Travassos, L. R.; Sartorelli, P.; Lago, J. H. G.; Biochem. Biophys. Res. Commun. 2011, 411, 449; Mosmann, T.; J. Immunol. Meth. 1983, 65, 55.

17. Lua, J. J.; Weib, Y.; Yuan, Q. P.; Sepr. Purif. Technol. 2007, 55, 40; The Merck Index; $9^{\text {th }}$ ed., Merck and Co. Inc.: Rahway, 1976; Ma, X.; Wu, L.; Ito, Y.; Tian, W.; J. Chromatogr., A 2005, 1076, 212.

18. Ayres, M. C. C.; Chaves, M. H.; Rinaldo, D.; Vilegas, W.; Vieira Júnior, G. M.; Quim. Nova 2009, 32, 1509.

19. Agrawal, P. K.; Bansal, M. C.; Porter, L. J.; Foo, L. Y. Em Carbon-13 NMR of Flavonoids - Studies in Organic Chemistry 39; Agrawal, P. K., ed.; Elsevier Science Publishers B.V.: Amsterdam, 1989.

20. Kaur, M.; Velmurugan, B.; Rajamanickam, S.; Agarwal R.; Agarwal, C.; Pharm. Res. 2009, 26, 2133; Yoshioka, K.; Kataoka, T.; Hayashi, T.; Hasegawa, M.; Ishi, Y.; Hibasami, H.; Oncology Rep. 2000, 7, 1221; Saleem, A.; Husheem, M.; Härkönen, P.; Pihlaja, K.; J. Ethnopharmacol. 2002, 81, 327. 